\title{
Editorial
}

\section{La cuestión ambiental: de la razón a las cosas del corazón}

\author{
Claudio Aguayo, PhD
}

Centre for Learning and Teaching, Auckland University of Technology

Quisiera invitarlos a reflexionar sobre tres puntos que considero claves al abordar el tema de las perspectivas y desafíos de la educación en ciencias experimentales, naturales, matemática y tecnología, en la integración de las cuestiones ambientales. Estos son: el foco de atención cuando nos referimos a las cuestiones ambientales; la identidad valórica de las ciencias en distintos contextos socioculturales; y la dimensión emocional y afectiva asociada a la temática ambiental. Estos puntos son claves por su potencial en la integración de la educación de las ciencias con el tipo de empoderamiento que permita a la ciudadanía responder y resolver sus conflictos ambientales, basado en las competencias y conocimientos requeridos para las transformaciones locales hacia la sustentabilidad socioecológica.

Para comenzar, me parece que podemos apuntar a la presencia de una mala costumbre, o más bien un problema terminológico y de enfoque que puede llegar a tener implicancias profundas, cuando nos referimos a las cuestiones ambientales. La temática ambiental a la que nos referimos hoy en día surge hacia fines de la década de 1960 y principios de la de 1970, como una inquietud transversal y global basada en el evidente deterioro del medio ambiente natural. Muchos son los que identifican el libro de Rachel Carson Silent spring (1962) como el primer gran llamado de atención a las posibles calamidades medioambientales que vemos en la actualidad —aunque Aldo Leopold (1949) hablaba de la ética de la Tierra y la conciencia ecológica antes que Carson-.

El foco de atención inicial era, en ese entonces, el medioambiente natural. Esto se hace evidente en la dinámica de la época en torno a la problemática ambiental, como queda establecido, por ejemplo, en la Carta de Belgrado de 1975 y en la Declaración de Tbilisi de 1977, ambas instituidas por la Organización de las Naciones Unidas para la Educación, la Ciencia y la Cultura, en conjunto con el Programa de Naciones Unidas para el Medio Ambiente (UnesCO-UNEP, 1976, 1978).

Sin embargo, a partir de los años 1980 podemos ver un cambio en el discurso global, reconociendo como sociedad contemporánea que la temática ambiental no solo concierne estrictamente al medio ambiente natural, sino que también se relaciona con otras dimensiones, sobre todo la económica y social. 
Así, durante las décadas del ochenta y noventa, las concepciones transitan desde la temática ambiental relacionada con el medioambiente natural, a un significado del ambiente como una totalidad (abarcando lo social, ecológico y natural), a más recientemente el concepto de sustentabilidad, caracterizado por enfocarse en la integración de las dimensiones social, económica y ambiental. El orden de estas y cuál contiene a cuál es muy relevante: algunos ven el medioambiente y la sociedad como un subsidiario de la economía, otros defienden lo opuesto.

En esta transición desde lo ambiental/natural a lo sustentable se transita hacia lo socioecológico, quizás como una forma de evidenciar dicha totalidad asociada al concepto más contemporáneo de medio ambiente. Esto también tal vez ocurre de la mano de una transición ontoepistemológica paralela en el discurso global durante los últimos cincuenta años en cuanto a la cuestión ambiental, que va en principio de lo cuantitativo/positivista, a lo cualitativo/naturalista, hasta lo complejo y sistémico. El punto clave aquí es que dicha transición y evolución terminológica en sí apunta a la expansión de lo que a principios se entendía abarcaba lo meramente ambiental/natural, donde el medioambiente era visto como algo externo al individuo como sujeto, y propio del medio natural, hacia un concepto de medio ambiente visto como interconectado e integral al sujeto. Por lo mismo, las cuestiones ambientales vistas como algo externo al individuo y meramente asociadas al medio natural, pasan a ser algo de lo que formamos parte íntegra a través de una red de relaciones complejas e impredecibles.

La mala costumbre, o el problema terminológico y de enfoque (ontoepistemológico), está en que hoy deberíamos estar hablando de las cuestiones ambientales como cuestiones de sustentabilidad, y ojalá con el apellido socioecológica. Un ejemplo de las implicancias que esto puede tener es lo que ocurre en Chile. La interrupción del desarrollo natural de una escuela local en educación ambiental debido al gobierno militar (1973-1989) (Squella, 2001), ha generado que en la actualidad, en ese país, la cuestión ambiental sea aún tratada desde el punto de vista ambiental/natural. Esto se evidencia en la semiótica del mensaje ambiental en los medios; por ejemplo, en las redes sociales del Ministerio del Medio Ambiente de Chile donde cuando se habla de medioambiente o sustentabilidad estos conceptos suelen relacionarse con paisajes lindos y animales de la fauna local. ¿ Cuáles serían las implicancias, en este caso concreto, si en vez de hablar de las cuestiones ambientales estuviésemos enfocándonos y enfatizando las cuestiones socioecológicas, particularmente en la educación de las ciencias experimentales y naturales, matemática y tecnología? Este interrogante surge de observar las publicaciones en Facebook del Ministerio del Medio Ambiente de Chile. Tal vez un pequeño cambio terminológico podría traer consigo una gran transformación cultural y de emancipación social, como un punto palanca (leverage point), donde un pequeño cambio de configuración puede generar un gran cambio en un sistema (Meadows, 1999). Como se dice por ahí: las palabras conllevan los significados, y los significados crean el mundo. 
Si es que el término cuestión ambiental se encuentra tan arraigado que es prácticamente inamovible, o es usado de manera tal que se subentiende el medio ambiente como una totalidad que incluye íntegramente lo 'sustentable' y lo 'socio-ecológico' escondido detrás del término 'ambiental' (Wals, 2007), deberíamos por lo menos ser muy claros cuando hablamos y enseñamos sobre la cuestión ambiental en ciertas audiencias no tan conocedoras del trasfondo semiótico. Esta perspectiva de que estamos íntegramente relacionados con nuestro medioambiente (ya sea social, económico o ambiental, u otros), y que lo que ocurra en cualquiera de estas dimensiones nos afecta directa o indirectamente sí o sí, y viceversa, es clave al integrar la educación de las ciencias con las cuestiones ambientales y el empoderamiento social. Por lo mismo, es importante ser claros en el mensaje y en la pedagogía asociada a la cuestión ambiental cuando queremos educar individuos y la ciudadanía en lo socioecológico, y así evitar confusión y malas interpretaciones, sobre todo a nivel de público general y educación no formal de comunidades.

Desde esta perspectiva socioecológica, al educar sobre cómo cuidar, proteger y preservar nuestro medioambiente nos estamos cuidando a nosotros mismos como especie que forma parte del puzzle ecosistémico. Y aquí radica una idea fundamental en esta discusión, no estamos conectados a nuestro medio ambiente, sino más bien somos el medio ambiente. Basta con imaginarse las implicancias que podría tener esta visión en la articulación de la educación de las ciencias en la integración de las cuestiones ambientales. Esta idea surge desde lo denominado "Santiago School of Cognition" (Hallowell, 2009), una reflexión surgida de los trabajos tempranos de Humberto Maturana y Francisco Varela (1980)

Estas ideas nos llevan al segundo punto clave: si bien las ciencias naturales, experimentales, y la matemática pueden ser, y son, consideradas ciencias puras, en el sentido que en su origen y formulación teórica no son provistas de una identidad valórica, su aplicación en distintos contextos socioculturales sí incorporan un sesgo valórico. Las prácticas educativas y de enseñanza, y su correspondencia con las características y las necesidades reales de cada contexto, conllevan un componente histórico, social, cultural, educacional, tecnológico, político, y económico (entre otros). Estos componentes (o dimensiones) configuran el marco valorativo de orden local y el tipo particular de educación de las ciencias desarrollado o a desarrollar, generando un cambio en los niveles de integración de las cuestiones ambientales con los desafíos socio ecológicos locales, y viceversa.

Más allá de tratar de abordar y entender cómo dichas dimensiones interactúan entre sí, y cómo pueden afectar los distintos procesos de aprendizaje y de integración de las cuestiones ambientales, cabe resaltar que es crucial a la hora de promover un mundo socio ecológicamente más sustentable, a través de la educación en general, y de la educación de las ciencias en particular, el cómo entendemos y practicamos nosotros como educadores la importancia de 
tomar en cuenta dichas dimensiones definidas localmente, y cómo este proceso en la práctica impacta en el tipo de educación y aprendizaje que queremos y podemos proveer.

Bajo esta mirada (ontológicamente naturalista) se puede decir que cada contexto histórico y sociocultural es único en el espacio/tiempo. Esto implica que lo que pueda llegar a ser relevante y significativo en un determinado contexto, o el tipo de práctica educativa que funciona en dicho contexto, no necesariamente sea aplicable en otro contexto similar. Por lo mismo, en el ámbito de la educación y emancipación de individuos y comunidades en las cuestiones ambientales es vital entender, comprender y adaptarse a las necesidades y particularidades socioculturales de cada contexto. Sobre todo en lo que corresponde a entender los problemas y conflictos socioecológicos que puedan allí existir, y cómo a través de la educación de las ciencias, y la tecnología, se puede contribuir al desarrollo de una cultura socioecológica y de empoderamiento social local y global (Aguayo, 2015).

Es muy llamativo ver que los artículos presentes en esta edición de TED, de alguna manera $u$ otra, hacen hincapié, o reflexionan en torno a la articulación de las temáticas científicas con las realidades locales sociales, y con temáticas culturalmente relevantes que contextualizan así la enseñanza de las ciencias a través de miradas más cualitativas y socialmente prácticas y tangibles. Por ejemplo, el artículo de Ferreira y Pereira, quienes abordan el tema de la crisis hídrica en Brasil desde la enseñanza de las ciencias, con estudiantes de sexto año en una escuela de Curitiba, donde se aborda la problemática del recurso agua en Brasil y el mundo al nivel sociocultural en pro de promover discusiones profundas entre los estudiantes. $\bigcirc$ el escrito de Yair Porras quien se centra en el reconocimiento de las representaciones sociales ligadas a la crisis ambiental en un grupo de profesores en formación de la Licenciatura en Química en Colombia. O el caso que presenta Leidy Bravo y la investigación realizada con pobladores del río Caquetá (Amazonia colombiana), y la relación entre sus discursos y las prácticas asociadas al ambiente en el contexto del manejo integral de humedales y el recurso pesquero de la cuenca de dicho río. O el artículo de William Manuel Mora, quien invita a reflexionar sobre la educación en ciencias y la formación ambiental del profesorado, a la vez que recalca la necesidad de reconceptualizar las competencias científicas hacia aquellas competencias ambientales claves, que permitan el desarrollo de estrategias didácticas sustentadas por las cuestiones socialmente vivas.

En este sentido, pareciera que la articulación de las cuestiones ambientales en la educación de las ciencias, matemáticas y tecnología, y las tensiones que puedan existir entre estos dos ámbitos, casi que se resuelven por sí mismo, o por su propio peso, desde la sociedad viva. Esta sociedad viva está urgida de capacidades específicas atingentes a las realidades locales que promuevan una adaptación y transformación social natural de las ciencias hacia dichas necesi- 
dades y el bienestar socioecológico de los individuos y sus comunidades locales. El desafío queda en nuestras manos como educadores; en tener la capacidad de ver, entender y adaptarnos a dichas particularidades de cada escenario; en comprender entender nuestras audiencias y cómo llegar a ellas de manera orgánica y natural, pudiendo así atender sus necesidades específicas de manera continua, a la vez de ser capaces de emancipar y empoderar.

Y esto nos lleva al tercer punto. Existe una dimensión poco explorada y explotada en educación ambiental (Payne et al., en preparación) que puede llegar a tener grandes implicancias a la hora de poder conectarnos con nuestras audiencias, y poder facilitar la articulación e integración de las cuestiones ambientales en la educación de las ciencias experimentales, naturales, matemática y tecnología: lo ecosomaestético y afectivo (Thrift, 2008), o en otras palabras, la dimensión emocional, sentimental, y las cosas del corazón.

La dimensión de lo emocional y lo afectivo, y el rol que puede llegar a tener en el aprendizaje de individuos y comunidades en la integración de las cuestiones ambientales con la educación de las ciencias nos plantea perspectivas y desafíos que pueden ir más allá de nuestra zona de confort. Preguntas como: ¿̇ómo educar y enseñar ciencias puras teniendo en cuenta las emociones y afectividades de individuos y comunidades? ¿Qué tipo de aproximaciones filosóficas y metodológicas requiere dicho proceso? ¿ Cómo podemos exaltar para bien dichas dimensiones a través de las ciencias y la tecnología en educación? ¿Deberíamos siquiera considerar tal tipo de integración entre las ciencias y lo sentimental? ¿Existen beneficios reales para la sustentabilidad socioecológica del planeta? ¿O tal vez para articular las cuestiones ambientales con el empoderamiento y emancipación social a través de la educación de las ciencias?

Lo que nos hace humanos en relación a lo tecnológico y material es lo carnal y visceral; los sentimientos, emociones y afectividades ecosomáticas, el amor y las cosas del corazón son tal vez los ingredientes que la sociedad viva pide a gritos para reconectarnos con nuestro medioambiente natural, con nosotros mismos, con el flujo armónico y sagrado de la vida y lo vivo, lo autoecopoiético y espiritual.

Como educadores podemos y debemos facilitar la integración y el acoplamiento de las cuestiones ambientales a la enseñanza y educación de las ciencias, con el fin de emancipar y empoderar a la ciudadanía hacia la sustentabilidad socioecológica. Existen varios caminos y fórmulas. Hoy en día la tecnología, por ejemplo, puede ser un gran aliado o, por el contrario, un enemigo de temer, depende de cómo moldeamos su uso a las características socioculturales específicas. Las ciencias naturales y exactas pueden contribuir a todas aquellas comunidades que saben y ven día a día cómo su entorno ecosistémico está en constante deterioro. Muchas veces dichas comunidades no tienen las bases científicas para tomar las decisiones y acciones necesarias para contrarrestar los efectos negativos con soluciones efectivas en el corto, mediano y largo plazo, y así salvaguardar sus recursos naturales cada día más escasos; recursos natu- 
rales que muchas veces también son las bases de la espiritualidad cultural muy presente en los pueblos indígenas de Latinoamérica.

Por eso, la educación de las ciencias experimentales y naturales, matemáticas y tecnología, debe promover un tipo de conocimiento ambiental y ecológico que sea atingente a la visión ecosistémica e integrada del medio ambiente; que pueda adaptarse e impregnarse de un discurso y un mensaje apropiado y vanguardista para cada trasfondo histórico y sociocultural, a la par de dirigir la educación hacia el empoderamiento y emancipación social; y saber cómo llegar al corazón de nuestros pueblos, de la sociedad viva y del continuo que existe entre nosotros y la carne del mundo (Merleau-Ponty, 1968), a través de un tipo de educación de las ciencias que apele a los sentimientos y exalte lo emocional, lo afectivo y lo ecosomaestético.

Si queremos influenciar y configurar la cuestión ambiental desde la educación de las ciencias de tal manera, debemos tener la facultad de transformarnos nosotros mismos como educadores, sentir y entender la sociedad viva cuando miramos hacia adentro de nosotros y exploramos la carne del mundo. Basta con imaginar las implicancias para la educación de las ciencias y las cuestiones ambientales, y aquí yace nuestro real desafío.

Nota: Quisiera agradecer a la Dra. Laísa Freire dos Santos por sus valiosos comentarios y aportes en la preparación de esta editorial.

\section{Referencias}

Aguayo, C. (2015). Activity theory and community education for sustainability: When systems meet reality. En: D. Gedera y J. Williams (eds.). Activity Theory in Education: Research and practice. (Chapter or section in book). Sense.

Carson, R. (1962). Silent spring. Cambridge, Mass.: Riverside Press.

Hallowell, R. (2009). Humberto Maturana and Francisco Varela's Contribution to Media Ecology: Autopoiesis, The Santiago School of Cognition, and Enactive Cognitive Science. En: P.A. Soukup (Ed.). Proceedings of the Media Ecology Association (pp. 143-158). Saint Lovis, MO: Media Ecology Association.

Jensen, B.B. y Schnack, K. (1997). The Action Competence Approach in Environmental Education. Environmental Education Research 3(2), 163-178.

Leopold, A. (1949). A sand county almanac. Nueva York, NY: Oxford University Press. Maturana, H.R. y Varela, F.J. (1980). Autopoiesis and cognition. The realization of the living. Dordrecht, Boston, Londres: D. Reidel Publishing Company.

Meadows, D. (1999). Leverage points: Places to intervene in a system. Hartland, VT: The Sustainability Institute.

Merleau-Ponty, M. (1968). The visible and the invisible. Evanston: Northwestern University Press. 
Orr, D. (1992). Ecological literacy: Education and the transition to a postmodern world. Nueva York: State University of New York.

Payne, P.; Rodrigues, C.; Carvalho, I.; Freire, L.; Aguayo, C. y lared, V. (2016). Affectivity in environmental education research. Pesquisa em Educação Ambiental. Sin publicar.

Squella P., M.P. (2001). Environmental Education to Environmental Sustainability. Educational Philosophy and Theory 33(2), 217-230.

Thrift, N. (2008). Non-representational theory: Space, politics, affect. Londres: Routledge.

Unesco-Unep (1976). The Belgrade Charter. Connect I(1), 1-10.

Unesco-UnEP (1978). The Tbilisi declaration. Unesco-UNEP Environmental Education Newsletter. Connect III(1), 33-40.

Wals, A.E.J. (2007). Learning in a Changing World and Changing in a Learning World: Reflexively fumbling towards sustainability. Southern African Journal of Environmental Education, 24, 35-45. 